Editorial

\title{
What makes for a good innovation management article?
}

\author{
Marko Torkkeli $^{1}$, Anne-Laure Mention ${ }^{2}$, João José Pinto Ferreira ${ }^{3}$ \\ ${ }^{1}$ Lappeenranta University of Technology, Finland; ${ }^{2}$ RMIT University, Australia; ${ }^{3}$ INESC TEC - \\ INESC Technology and Science and FEUP - Faculty of Engineering, University of Porto, \\ Portugal \\ marko.torkkeli@lut.fi, anne-laure.mention@rmit.edu.au, \\ jjpflefe.up.pt
}

What makes a good innovation management article? Let's have a look at articles which incidentally have got the highest citations in the field of innovation management. These articles may give us some ideas on what a good innovation management publication entails. Notions below are not fully covering what should be included in article from introduction to final words; it is more about suggestions based on our cumulative experience in the field and some general observations as editors.

Most articles typically highlight some points within generally held assumptions in innovation management current literature. Extensive literature review is important for all papers, and here authors may go even beyond usual suspects. By expressing this, we mean multidisciplinary approaches on innovation where knowledge should flow from different sources into your innovation storyline. Most articles are not written using a T-shaped knowledge model initially discussed by IBM, but still tell us the story of innovation management in the broader context and allow us broadening our scope in the field. The Journal of Innovation Management is not just a new venue for publishing innovation related articles. We implicitly explained in our inaugural editorial that the Journal of Innovation Management concentrates on the combination and integration of horizontal perspective of innovation management into vertical perspective where we go deeply into the specific silos of highly technological knowledge. This is often tackled superficially in innovation management papers, unfortunately.

The connection between the results and conclusion needs to be carefully explained. Where the innovation lies in your story that explains carefully your conclusions made? One way to confirm is to ask yourself whether the outcome would be the same without innovation management element. By definition, innovation creates value and well-being, so in most cases, it means novelty with commercialization in the market.

Several articles showcase well known global corporations and their innovation management protocols. There is nothing wrong here, but the question is how you express the novelty. We are not only looking for well-known examples about companies which have been successful in the market for years and have been well explained already or well-known by the big audience in the market. These big companies should be used as an example as long as the innovation storyline behind 
can be in reliable and explain explicitly what leads to the results and conclusions. The reasoning between connecting these two dots is not easy to tell as there are many other factors which can have an influence and typically, these are external factors that we cannot control internally when we explain our innovation management. For example, by 2017, central banks in EU, US, China and Japan have done quantitative easing by pushing more money to the market than ever in our history which have strong influence on stock valuations and consumer markets. It is definitely an external factor of innovation story and extremely difficult to connect to your innovation management storyline of a company.

Topical issues are interesting in general for a broad audience. Trend is your friend and one potential arena could be on how profits are generated and shared in a specific industry. For example, in the mobile phone industry in 2016, the leading player took over $80 \%$ of profits where hundred other companies barely managed to reach break even. Value generation seems to be easy for engineers, but value capturing is not that easy as it takes more than technological breakthrough. Teece (1986) introduced appropriability regime which may help us to understand and analyze the situation. And there are other industries as well like online marketing to name one. Here we may propose a 'new' pareto principle (original introduced by management consultant Muran in 1940s, named after Italian economist Vilfredo Pareto): instead of 80/20 we may say $20 / 60 / 20$ where 20 percent of market population is extremely profitable, following $60 \%$ makes some profits in good times and final $20 \%$ make losses independently of market conditions. Does this make sense in several industries (mostly mature ones)?

Finally, we should not avoid conducting research and presenting stories of failures. We acknowledge that it is difficult to collect data about failures, or at least when company information is revealed, but still from a learning perspective we need those. So here upon we call for stories about failures - for public good and lessons to be learned!

Innovatively Yours,

Marko Torkkeli, Anne-Laure Mention, João José Pinto Ferreira

Editors

\section{References}

Teece, D.J. (1986), "Profiting from technological innovation: Implications for integration, collaboration, licensing, and public policy," Research Policy. 